\title{
Post salpingectomy intraluminal endometriosis in a premenopausal lady - an incidental finding often paid less attention to
}

\author{
Kanna Sandhyarani, Archana Shetty*, Bhargavi Kalburgi Nagabhushan, \\ Aparna Muralidhar, Jessica Minal, Nikhil Moorchung
}

\begin{abstract}
Department of Pathology, Dr. Chandramma Dayanada Sagar Institute of Medical Education and Research, Kanakapura
\end{abstract} Road, Ramanagara District, Karnataka, India

Received: 10 January 2021

Accepted: 10 February 2021

\section{*Correspondence:}

Dr. Archana Shetty,

E-mail: archanashetty2924@gmail.com

Copyright: (C) the author(s), publisher and licensee Medip Academy. This is an open-access article distributed under the terms of the Creative Commons Attribution Non-Commercial License, which permits unrestricted non-commercial

\begin{abstract}
Endometriosis of the fallopian tube is often incidentally picked up in hysterectomy specimens that are sent for histopathological examination for other obvious pathological conditions. Post-salpingectomy endometriosis is one such entity that is known to occur in the tip of the proximal stump of the fallopian tube years after tubal ligation. As mere visualization of the endometriotic lesions is inadequate for an accurate diagnosis, histopathologic analysis of the biopsy samples becomes mandatory for confirmation. We report a case of post salpingectomy endometriosis which was incidentally discovered in a peri menopausal lady who was operated for multiple fibroids of the uterus. This case not only highlights an entity which is challenging to visualize radiologically and suspect clinically, but is also underrecognized, as very little attention is given to the fallopian tube during routine grossing.
\end{abstract}

Keywords: Post salpingectomy, Endometriosis, Tubal ligation, Histopathology

\section{INTRODUCTION}

Non-neoplastic lesions of the fallopian tubes often give rise to diagnostic challenges to the unaware, as many of them are uncommon. ${ }^{1}$ Postsalpingectomy endometriosis which can be broadly divided into serosal or intraluminal, commonly occurs 1-4 years after tubal ligation, with the patients presenting with nonspecific symptoms. ${ }^{2,3}$ Intraluminal endometriosis is less common and involves ectopic implantation of endometrium on the mucosal surface of the tube lumen.

Cyclic hemorrhage of the implants can cause distention of the fallopian tube with blood (ie, hematosalpinx). ${ }^{2}$ However tubal endometriosis, specially the intraluminal type is often not visualized on imaging and is incidentally diagnosed when the tubes are subjected to histopathological examination for other symptomatic pathologies. $^{2}$

\section{CASE REPORT}

A forty-six-year-old female was diagnosed with anaemia and fibroid uterus when she presented to the gynaecological OPD of our hospital with complaints of abnormal uterine bleeding. USG abdomen showed multiple fibroids. The patient was admitted, a total laparoscopic hysterectomy with bilateral salpingectomy was done and the specimen was sent in $10 \%$ formalin for histopathological examination.

On gross examination, the uterus- cervix measured $13 \times 8 \times 4 \mathrm{~cm}$. External surface of uterus appeared irregular and showed a subserosal fibroid measuring $2 \times 2 \mathrm{~cm}$ across. Cut surface of uterus showed multiple intramural fibroids distorting the endometrial cavity, largest measuring $3 \mathrm{cms}$ in diameter and smallest measuring 0.5 $\mathrm{cm}$, all with pearly white whorled appearance. Cervix showed Nabothian cysts. One of the attached fallopian tube measured $5 \mathrm{cms}$ and the other $7.5 \mathrm{~cm}$, with both 
tubes showing evidence of pomeroy type of tubectomy without cautery marks. The smaller tube showed evidence of tubal ligation proximal to which the thickened wall and narrowed lumen with brownish discoloration was noted. (Figure 1) The other tube was unremarkable.

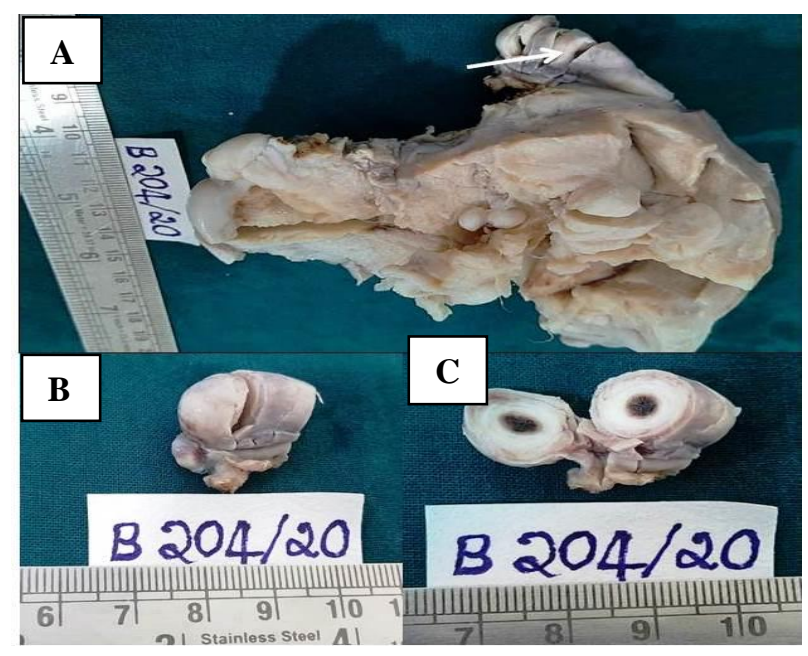

Figure 1: (A) Cut section of the hysterectomy specimen showing multiple fibroids. (B) Thickened

Proximal stump of the fallopian tube. (C) Cut section of the tube showing wall thickening, and luminal narrowing with brownish discoloration.

Microscopy of the shorter tube proximal to the ligation showed the tubal plicae to be completely replaced by endometrial type of tissue comprising of glands and stroma (Figure 2). The glands were tortuous and lined by tall columnar pseudo stratified epithelium (Figure 2). Stroma showed oedema, haemorrhage and foci of chronic inflammatory infiltrate. The tubal wall showed muscular hyperplasia with congested serosal blood vessels. All these features were consistent with endometrialisation of tube post ligation.

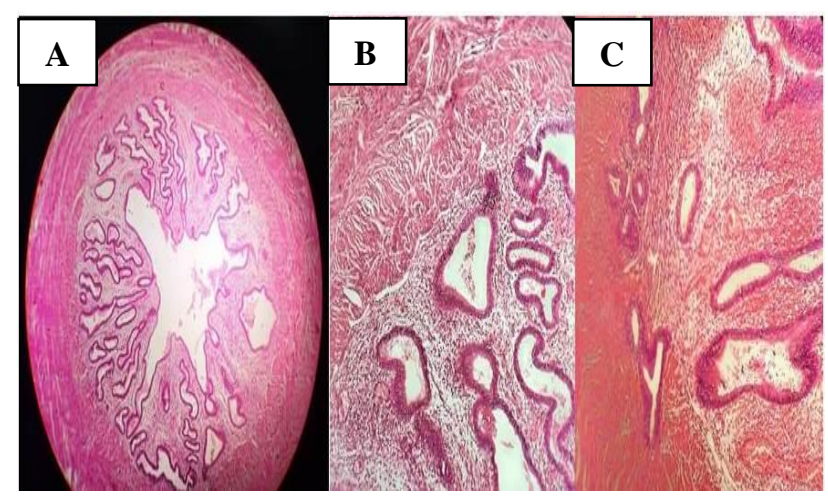

Figure 1: Microphotographs showing (A) lumen of the fallopian tube completely replaced by endometrial tissue (B) dilated and tortuous endometrial glands (C) intervening stroma showing areas of haemorrhaage $\mathbf{H}$ and E stain.
The tubal portion distal to ligation were histologically normal in both tubes. Endometrium showed late proliferative phase. Myometrium showed leiomyomata and cervix showed features of chronic cervicitis with Nabothian cysts.

\section{DISCUSSION}

Tubal endometriosis is a rarely encountered type of endometriosis compared to other subtypes. The pathogenesis and initiation of this lesion is complex. ${ }^{4}$ Its clinical manifestation is poorly documented and diagnosis is frequently incidental after hysterectomies for other indications like fibroids, endometrial hyperplasia and adenomyosis. ${ }^{5}$ The diagnosis in our case was also made incidentally while sampling the fallopian tubes sent in a hysterectomy done for a patient who was operated for multiple fibroids.

The standard textbooks and the literature referenced here all mention three types of tubal endometriosis- firstserosal (most common type encountered), Second-the normal morphologic variation also termed as endometrial colonisation, which is seen in isthmic and interstitial part of fallopian tube and Third- post salpingectomy endometriosis. ${ }^{6}$ The latter two are under recognised types possibly due to 1) lack of knowledge and awareness among pathologists about them 2) The fact that they don't present as specific gynaecological diseases 3) sampling of fallopian tube in hysterectomies is more of a completion procedure and not a part of specific diagnosis as otherwise would be done when salpingectomy is specifically clinically indicated for conditions like hematosalphinx, torsion etc. in the pre-menopausal age group. The same opinion is shared by other authors who reported similar cases. ${ }^{1}$

Post-salpingectomy endometriosis occurs at the tip of the proximal tubectomy stump, years after the procedure, particularly with the use of electrocautery, short proximal stumps and with increasing post ligation intervals and at times is often related to salpingitis isthmica nodosa. ${ }^{1}$ Endometriosis is an estrogen driven disease and progesterone resistance is considered to be a crucial requirement for the seeding and maintenance of endometriotic lesions. ${ }^{4}$ However the tubal mucosa is known to be less hormonally responsive than the neighbouring endometrium and may not exhibit similar cyclical proliferative dynamics. ${ }^{4}$ In our case too there were no concordance between the endometrial phase and the tubal endometriotic tissue.

As with other types of endometriosis, the tubal endometriosis is also accompanied by inflammation which leads to functional disturbances and morphological changes in fallopian tube. ${ }^{7}$ One of the major reasons for endometriosis causing pain is the release of inflammatory mediators such as cytokines, chemokines and growth factors by the endometriotic lesions. ${ }^{8}$ The clinical 
implications of these is not well known in case of post salpingectomy endometriosis.

The differential diagnosis in our case was salpingitis isthmica nodosa. Postsalpingectomy endometriosis is often considered to be related to salpingitis isthmica nodosa. In this, the muscularis is greatly thickened with the tubal lumen apparently been split into numerous small canals scattered throughout the muscularis at various distances between the lumen proper and the serosa. ${ }^{1}$ The post tubectomy status, presence of features of endometrial type of tissue replacing the tubal lumen completely proximal to the ligation site were favouring a diagnosis of post salpingectomy endometriosis in our case.

The controversies exist over the origin of endometrial tissue in tubes. Sampson's metaplasia theory has been challenged by Stock, who was of the opinion that endometriosis of the proximal stump is a result of repeated menstrual reflux. ${ }^{9}$ There are interesting theories and extensive research which explain the role of inflammation, role of endometrial stem cells or tubal stem cells and varied hormone responsiveness of tubal epithelium in causation of tubal endometriosis. ${ }^{4}$

Overall, the post salpingectomy endometriosis or endometrialisation of tube post ligation is an entity the pathologists are frequently unaware (due to aforementioned reasons) and is not enough thought of as a research topic. We propose few important points as to why this diagnosis is important 1) if post salpingectomy cases are studied extensively, there may be an increase in prevalence, awareness and reporting of this entity 2) the data can be large enough to form a study model about the intriguing pathogenesis of tubal endometriosis 3) the procedure of sampling the fallopian tube can be paid more attention to-instead of processing a single cross section in hysterectomies, a longitudinal section of tubes can be studied.

This will not only help pick up the pathologic entities of non-neoplastic lesions of the fallopian tube which are less talked about but also help rule out the occult malignancies arising from the tubal epithelium, considering the increase in evidence of theory of origin of ovarian carcinomas from the tubal epithelium in recent literature. ${ }^{10}$

\section{CONCLUSION}

The case highlights the histopathologists the importance of an extensive sampling of the fallopian tube specially in post ligation hysterectomy cases and to go beyond just superficial grossing for organ documentation completion, which is the usual practice. An awareness of the morphological variations in tubal endometriosis may open new possibilities to customize suitable lesion type specific treatments in the future for the treating gynaecologists.

\section{ACKNOWLEDGMENTS}

We thank the faculty from the Dept of Obstetrics and Gynecology of our hospital for their support.

Funding: No funding sources

Conflict of interest: None declared

Ethical approval: Not required

\section{REFERENCES}

1. Ramaswamy A.S., Manjunatha H.K. Postsalpingectomy endometriosis. J Lab Physician 2009;1(2):84-5.

2. Rezvani M, Shaaban AM. Fallopian tube disease in the nonpregnant patient. Radiographics 2011;31(2):527-48.

3. Tandon RK, Pandya SP, Darad D, Chugh A, Bhuva K. Laparoscopic scar - laparoscopic tubal ligation: A rare case report. J Clin Diagn Res 2012;6(4):707-8.

4. Hill CJ, Fakhreldin M, Maclean A, Dobson L, Nancarrow L, Bradfield A et al. Endometriosis and the Fallopian Tubes: Theories of Origin and Clinical Implications. J Clin Med 2020;9(6):1905.

5. Xue RH, Li J, Huang Z, Li ZZ, Chen L, Lin Q et al. Is tubal endometriosis an asymmetric disease? A 17year retrospective study. Arch Gynecol Obstet 2020;301(3):721-7.

6. Chakrabarti I., Ghosh N. Post-salpingectomy endometriosis: An under-recognized entity. J Midlife Heal 2010;1(2):91-2.

7. Xia W, Zhang D, Ouyang J, Liang Y, Zhang H, Huang $Z$ et al. Effects of pelvic endometriosis and adenomyosis on ciliary beat frequency and muscular contractions in the human fallopian tube. Reprod Biol Endocrinol 2018;16(1):48.

8. Garcia-Velasco JA. Fallopian tube endometriosis: clinical implications. Fertil Steril 2020;114(5): 966.

9. Stock RJ. Histopathologic changes in fallopian tubes subsequent to sterilization procedures. Int J Gynecol Pathol 1983;2:13-27.

10. Magdy N, El-Bahrawy M. Fallopian tube: Its role in infertility and gynecological oncology. World $\mathrm{J}$ Obstet Gynecol 2014:3(2):35-41.

Cite this article as: Sandhyarani K, Shetty A, Bhargavi KN, Muralidhar A, Minal J, Moorchung N. Post salpingectomy intraluminal endometriosis in a premenopausal lady - an incidental finding often paid less attention to. Int $\mathbf{J}$ Reprod Contracept Obstet Gynecol 2021;10:1221-3. 